\title{
Complete resolution of chylothorax with ibrutinib in chronic lymphocytic leukemia: a case report
}

\author{
Ling Cheng ${ }^{1 \#}$, Mei Huang ${ }^{2 \#}$, Jia Wei ${ }^{2}$, Hao Xu' ${ }^{2}$, Mi Zhou ${ }^{2}$, Lijun Jiang ${ }^{2}$ \\ ${ }^{1}$ Department of Geriatrics, Tongji Hospital, Tongji Medical College, Huazhong University of Science and Technology, Wuhan, China; ${ }^{2}$ Department \\ of Hematology, Tongji Hospital, Tongji Medical College, Huazhong University of Science and Technology, Wuhan, China \\ "These authors contributed equally to this work. \\ Correspondence to: Lijun Jiang. Department of Hematology, Tongji Hospital, Tongji Medical College, Huazhong University of Science and \\ Technology, Jiefang Road 1095\#, Wuhan 430030, China. Email: 278308418@qq.com.
}

\begin{abstract}
Chylothorax is an infrequent pleural effusion often caused by traumatic or nontraumatic injury to the thoracic duct. Nontraumatic chylothorax caused by chronic lymphocytic leukemia (CLL) is rarely reported. Previous experience has implied that the main factor affecting the treatment of chylothorax is whether the anti-cancer treatment is effective. The patient diagnosed with chronic lymphocytic leukemia/ small lymphocytic lymphoma (CLL/SLL) before the hospital admission, and not received any treatment. After four months, he had progressive dyspnea. Chest computed tomography (CT) scan showed bilateral pleural effusion with progressive lymphadenopathy, and massive chylous pleural effusion was drained by closed thoracic drainage. After a course of chemotherapy including fludarabine, cyclophosphamide and rituximab (FCR), the patient developed agranulocytosis and his pleural effusion was still abundant. After careful consideration, the patient refused to receive following chemotherapy and chose to take ibrutinib orally. Two months after oral ibrutinib, ultrasound examination showed that pleural effusion completely disappeared. In the next one year, the patient had a routine follow-up and was in good condition. To our knowledge, this is the first report of ibrutinib in the treatment of chylothorax associated with CLL. Ibrutinib provides a more palliative treatment for elderly CLL patients with chylothorax.
\end{abstract}

Keywords: Chylothorax; chronic lymphocytic leukemia (CLL); ibrutinib; case report

Submitted Apr 22, 2021. Accepted for publication Jun 11, 2021.

doi: 10.21037/apm-21-989

View this article at: https://dx.doi.org/10.21037/apm-21-989

\section{Introduction}

Chylothorax is an infrequent pleural effusion often caused by traumatic or nontraumatic injury to the thoracic duct (1). Dyspnea and chest discomfort are often the first onset symptoms of chylothorax patients (2). Nontraumatic chylous pleural effusion is uncommon and often accompanied by malignant tumours, which most common in adult is lymphoma $(3,4)$. Other hematologic diseases, including chronic lymphocytic leukemia (CLL), are rarely reported. Up to now, the mechanism and treatment of chylous exudation are not clear. Effective chemotherapy is conducive to the spontaneous absorption of pleural effusion, which may solve the problem of chylothorax associated with hematological malignancies $(4,5)$.

Chronic lymphocytic leukemia/small lymphocytic lymphoma (CLL/SLL), commonly seen in adults, is an inert B-cell non-Hodgkin's lymphoma (NHL) (6). CD5-positive monomorphic round B lymphocytes, accumulated in bone marrow and blood (CLL), or limited in lymph nodes (SLL), are characterized by a distinctive immunophenotypic profile, co-expressing CD19, CD20, CD23 and other B cell surface antigens. CLL can infiltrate any organ, but chylous pleural effusion is very rare, which may be associated with rare mediastinal lymph node lesions (7). Over the past decades, regimen including fludarabine, cyclophosphamide and rituximab (FCR) has been the standard treatment for CLL patients. In recent years, with the development of new 

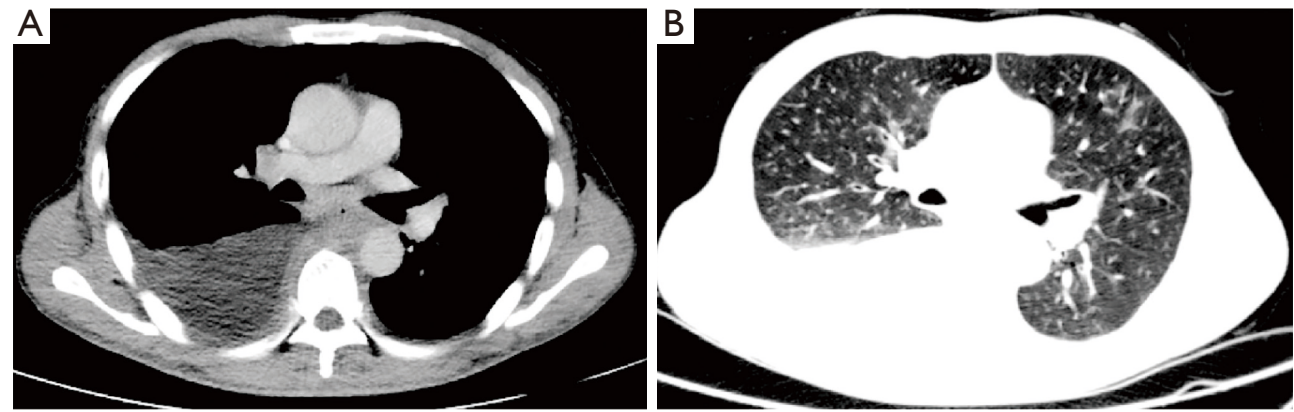

Figure 1 The results of pulmonary computed tomography (CT) scan show abnormalities in the lungs. (A) On the lung window image and (B) the longitudinal window image of the pulmonary CT scan, bilateral pleural effusion, especially on the right side, and bilateral lower lobe atelectasis.

targeted therapy drugs, such as inhibitor of Bruton tyrosine kinase (BTK), BCL2 inhibitors and anti-CD20 antibodies, the treatment of many high-risk or older CLL patients has become more effective and challenging than ever before $(6,8)$. Among these targeted drugs, ibrutinib, a first-in-class oral inhibitor of BTK, has been attracted more attention. To date, whether ibrutinib can treat chylothorax associated with CLL has not been reported. Herein, we describe a case of chylothorax in an elderly CLL patient without any previous treatment. He had progressive dyspnoea and was treated with closed thoracic drainage for massive pleural effusion. After a course of FCR chemotherapy, his chylous pleural effusion had a moderate reduction, but still existed. The patient developed grade III agranulocytosis and refused to receive following chemotherapy. After careful consideration, the patient chose to treat with ibrutinib. Two months after oral administration of ibrutinib, ultrasound examination showed that pleural effusion completely disappeared. In the next few months, the patient had a routine follow-up and was in good condition. We present the following case in accordance with the CARE reporting checklist (available at https://apm.amegroups.com/article/ view/10.21037/apm-21-989/rc).

\section{Case presentation}

A 67-year-old Chinese man presented with worsening dyspnea and dry cough on January 2020 and admitted to Tongji hospital. Reviewing the history, the patient was diagnosed with CLL/SLL four months prior to this hospitalization without any treatment. Upon review of the family history of the patient, no special medical history was found. Physical examination showed multiple enlarged lymph nodes in bilateral axillary and clavicle, without pain. A white blood cell count was $14.38 \times 10^{9} / \mathrm{L}$ with $73.2 \%$ lymphocytes. Hemoglobin was $128 \mathrm{~g} / \mathrm{L}$, and platelets were $103 \times 10^{9} / \mathrm{L}$. Hepatitis B core antibody (anti$\mathrm{HBc}$ ) and hepatitis B surface antibody (anti-HBs) were positive, while hepatitis B surface antigen (HBsAg) was negative. Furthermore, the amount of HBV-DNA in the serum was detected at very low level $(200 \mathrm{IU} / \mathrm{mL})$. Chest computed tomography (CT) showed bilateral pleural effusion, especially on the right side, and bilateral lower lobe atelectasis (Figure 1). In addition, CT images showed that lymph nodes in mediastinum, bilateral axillary and supraclavicle and retroperitoneal were increased and enlarged. The patient had no history of tuberculosis, alcohol or tobacco consumption, without symptoms of fever, hemoptysis, pneumonia or weight loss.

In view of progressive dyspnea and massive pleural effusion, our patient was treated with closed thoracic drainage immediately. Thoracic drainage revealed a chylous effusion. Cytologic analyses showed a large number of mature lymphocytes and no typical malignant cells. Flow cytometry analysis of the pleural effusion revealed 25.2\% B lymphocytic cells, monoclonal B cell immunophenotype CD5+, CD19+, CD20+, CD10-, CD43+, CD23+. Bone marrow biopsy was performed, and the results of cytology and flow cytometry of bone marrow were consistent with CLL. Subsequently, the results of karyotype analysis and fluorescence in situ hybridization showed no abnormality.

Combined with the patient's medical history, FCR chemotherapy, including fludarabine, cyclophosphamide and rituximab, was given at the first time. Prior to chemotherapy, our patient began to use anti HBV drugs due to occult hepatitis B virus Infection (anti-HBc antiHBs were positive) (9). Meanwhile, hundreds of milliliters pleural effusion was drained continuously every day. After 

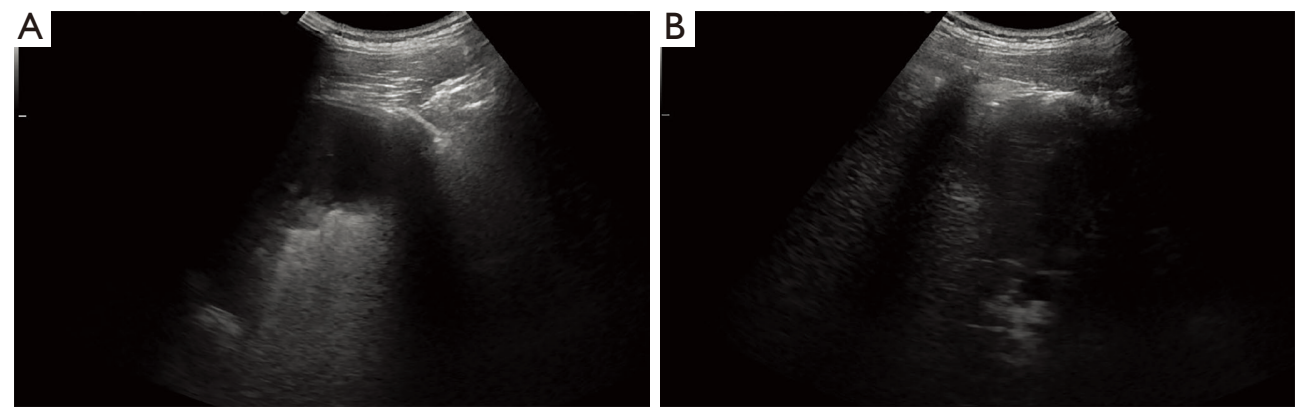

Figure 2 The examinations of B-ultrasound show pleural effusion of thoracic cavity. No pleural effusion was showed by B-ultrasound on the right side (A) and the left side (B) of thoracic cavity.

one course of chemotherapy, B-ultrasound indicated pleural effusion of our patient had a moderate reduction, but still existed. Unfortunately, the patient developed grade III agranulocytosis in the following days. After receiving hematopoietic growth factor support therapy, the number of white blood cells gradually recovered. However, our patient refused to receive any chemotherapy regimen again. Based on the persistent pleural effusion and after discussing with the patient and his family, ibrutinib was chosen for the patient. From March 2020, the patient took $420 \mathrm{mg}$ ibrutinib daily. On the 10th day since the beginning of oral ibrutinib, B-ultrasound indicated that pleural effusion was in decline, the drainage could be terminated and the patient was discharged. Two months after oral administration of ibrutinib, that pleural effusion of our patient was completely disappeared (Figure 2). In the next one year, the patient had a routine follow-up including routine blood test, HBsAg and HBV-DNA and B-ultrasound examination of thoracic cavity, but refused bone marrow aspirate/biopsy. The results showed that the blood routine was in the normal range, hepatitis $B$ virus was not activated, and there was no effusion in thoracic cavity.

All procedures performed in this study were in accordance with the ethical standards of the institutional and/or national research committee(s) and with the Helsinki Declaration (as revised in 2013). Written informed consent was obtained from the patient for publication of this case report and accompanying images. A copy of the written consent is available for review by the editorial office of this journal.

\section{Discussion}

Our manuscript introduced an untreated chronic lymphoblastic leukemia presenting with massive chylothorax. After one course of FCR chemotherapy, the pleural effusion was not significantly decreased. After careful consideration, the patient refused to receive next course chemotherapy and chose to ibrutinib orally. In the following two months, the pleural effusion gradually decreased until disappeared completely.

CLL is a lymphoproliferative disorder with strong inherited predisposition (10). About $10 \%$ of CLL cases report two or more individuals affected by CLL in the same family, which mainly occurs in western countries and has the same clinical and biological characteristics as sporadic cases. Upon review of the family history of our patient, no similar medical history was found in the family members.

There is a strong heterogeneity in the clinical process of CLL (11). The majority of patients follow a slow clinical process, often found in physical examination, no need or delay treatment. At the same time, there are also some patients with invasive disease requiring early treatment, and then followed by frequent recurrence. In general, the initiation of treatment depends on the Rai and Binet staging system and the presence of disease-related symptoms or not (10). Chylothorax, as a result of CLL, has only been reported in dozens of cases and rarely occurs during the late stage of the disease $(12,13)$. Four months before the visiting to us, our patient was found to have multiple lymphadenopathies in the whole body during a routine physical examination and diagnosed with CLL/SLL in other hospital. The patient received no treatment, and chylothorax occurred during the observation period. As shown in this case, flow cytometric analysis of pleural fluid showed that cell surface markers including CD3, CD5, CD19, CD20 were positive, consistent with CLL, which confirmed that the chylothorax in our patient was associated with leukemic infiltration $(10,13)$. The management of pleural effusion caused by hematological malignancies is challenging. Although previous experience has implied that auxiliary 
interventions, such as low-fat diet and parenteral nutrition, are important for the recovery of patients $(2,5)$. The main factor affecting the treatment of chylothorax is whether the anti-cancer treatment aimed at the primary disease is effective. Treatment of chylothorax usually lasts several months and is affected by the duration of chylothorax, the extent of chylothorax loss, and the general condition of the patient (14). Experiences from a retrospective study of chylothorax with lymphoproliferative disease showed that the average clearance time of chylothorax was 73 days after receiving anti-cancer treatment (5). For complex cases, other treatments besides chemotherapy should be considered. A case of refractory CLL with chylothorax was treated with different chemotherapy regimens, but the pleural effusion was not eliminated. Finally, irradiation of the thoracic duct was used. After 125 days of continuous treatment, chylothorax gradually absorbed in about one month after the end of irradiation (5).

In the first early decades, chloramphenicol has been used as a standard treatment for CLL. Subsequently, it was found that fludarabine combined with cyclophosphamide (FC) can obtain better response in younger CLL patients. Then, regimen include rituximab, the antiCD20 monoclonal antibody, has achieved good curative effect in CLL patients of all different ages (10). Thus, as the development of these drug combinations, rituximab plus FC (FCR) has created the first therapeutic regimen to prolong the overall survival of patients with chronic lymphoblastic leukemia. Due to the side effects of FCR such as bone marrow suppression, equally effective but less toxic therapeutic regimen has been attempted to be created. Therefore, bendamustine combined with rituximab can be used as an alternative first-line treatment to reduce potential toxicity for fit patients over 65 years old (15). But, the occurrence of bone marrow suppression or infection could not be avoided, prevention of infection or support of hematopoietic growth factors should be considered during treatment. Ibrutinib is a novel and potent oral drug and irreversibly inhibits BTK, a B-cell receptor (BCR) signaling kinase expressed by many hematopoietic cells, B-cell lymphoma and leukemia cells. Multiple clinical trials have shown that ibrutinib has a significant effect on CLL and is approved for treatment of CLL patient in any line of therapy. As an effective and well-tolerated treatment, ibrutinib has rapidly become the standard of care for patients with recurrent CLL and many first-line high-risk or elderly patients. In view of the discomfort caused by chemotherapy, our patient finally chose to oral ibrutinib after thorough consideration. Long term oral administration of ibrutinib will inevitably cause some side effects $(16,17)$. Cardiac complications are most common in elderly patients, especially atrial fibrillation. Ibrutinib-induced reactivation of hepatitis B has been reported. Although our patient with OBI started therapy before oral ibrutinib, he should be monitored closely in follow-up, including with HBV DNA testing. During ibrutinib treatment, attention also should be paid to the risk of bleeding or fungal infection and the influence of drug interaction (16). Meanwhile, arthralgia and myalgia, which were observed following long-term administration of ibrutinib, have been considered as a leading reason for discontinuation or dose adjustment in clinical practice (18).

Unfortunately, we did not fully evaluate the effect of FCR on patients in time. It is undeniable that the FCR regimen with multiple courses of treatment may achieve the same therapeutic effect. Given the general situation of the his body and the side effects caused by chemotherapy, the patient finally chose oral ibrutinib and achieved good therapeutic effect. After chylothorax disappeared, the patient remains under routine follow-up for about a year and is still in good condition.

To our knowledge, this is the first report of ibrutinib in the treatment of chylothorax associated with CLL. Ibrutinib provides a more palliative treatment for elderly CLL patients with chylothorax. In consideration of the heterogeneity of patients' response to treatment and the lack of relevant experience due to the rarity of the disease, choosing a suitable treatment regimen for CLL with chylothorax requires comprehensive consideration. We will continue to follow up the reports of CLL with chylothorax. The related cases will be further studied in order to better summarize the treatment experience.

\section{Acknowledgments}

We owe thanks to the patient and his family.

Funding: This work was supported in part by the Natural Science Foundation of Hubei Province (2019CFB656 to LJ Jiang) and the Youth Science Fund Project of NNSF of China (81800162 to M Zhou).

\section{Footnote}

Reporting Checklist: The authors have completed the CARE reporting checklist. Available at https://apm.amegroups. com/article/view/10.21037/apm-21-989/rc 
Conflicts of Interest: All authors have completed the ICMJE uniform disclosure form (available at https://apm. amegroups.com/article/view/10.21037/apm-21-989/coif). Mi Zhou reports receiving funding from Youth Science Fund Project of National Natural Science Fund of China (Number: 81800162). Lijun Jiang reports receiving funding from Natural Science Foundation of Hubei Province (Number: 2019CFB656). The other authors have no conflicts of interest to declare.

Ethical Statement: The authors are accountable for all aspects of the work in ensuring that questions related to the accuracy or integrity of any part of the work are appropriately investigated and resolved. All procedures performed in this study were in accordance with the ethical standards of the institutional and/or national research committee(s) and with the Helsinki Declaration (as revised in 2013). Written informed consent was obtained from the patient for publication of this case report and accompanying images. A copy of the written consent is available for review by the editorial office of this journal.

Open Access Statement: This is an Open Access article distributed in accordance with the Creative Commons Attribution-NonCommercial-NoDerivs 4.0 International License (CC BY-NC-ND 4.0), which permits the noncommercial replication and distribution of the article with the strict proviso that no changes or edits are made and the original work is properly cited (including links to both the formal publication through the relevant DOI and the license). See: https://creativecommons.org/licenses/by-nc-nd/4.0/.

\section{References}

1. McGrath EE, Blades Z, Anderson PB. Chylothorax: aetiology, diagnosis and therapeutic options. Respir Med 2010;104:1-8.

2. Majdalany B S, Murrey Jr D A, Kapoor B S, et al. ACR Appropriateness Criteria ${ }^{\circledR}$ chylothorax treatment planning. J Am Coll Radiol 2017;14:S118-26.

3. Romero S. Nontraumatic chylothorax. Curr Opin Pulm Med 2000;6:287-91.

4. Faiz SA, Pathania P, Song J, et al. Indwelling Pleural Catheters for Patients with Hematologic Malignancies. A 14-Year, Single-Center Experience. Ann Am Thorac Soc 2017;14:976-85.

5. Pospiskova J, Smolej L, Belada D, et al. Experiences in the treatment of refractory chylothorax associated with lymphoproliferative disorders. Orphanet J Rare Dis 2019;14:9.

6. Hallek M. Chronic lymphocytic leukemia: 2020 update on diagnosis, risk stratification and treatment. Am J Hematol 2019;94:1266-87.

7. Antón E. Chylothorax in hematologic malignancies. Chest 2005;127:1866-67.

8. Deeks ED. Ibrutinib: A Review in Chronic Lymphocytic Leukaemia. Drugs 2017;77:225-36.

9. Innocenti I, Morelli F, Autore F, et al. HBV reactivation in CLL patients with occult HBV infection treated with ibrutinib without viral prophylaxis. Leuk Lymphoma 2019;60:1340-2.

10. Hallek M, Shanafelt TD, Eichhorst B. Chronic lymphocytic leukaemia. Lancet 2018;391:1524-37.

11. Scarfò L, Ferreri AJ, Ghia P. Chronic lymphocytic leukaemia. Crit Rev Oncol Hematol 2016;104:169-82.

12. Morris AL, Colbourne T, Kirkpatrick I, et al. Complete resolution of chylopericardium after chemotherapy for chronic lymphocytic leukemia. Curr Oncol 2019;26:e696-9.

13. Tanriverdi H, Uygur F, Tilkan OK, et al. Chylothorax due to leukemic infiltration in a patient with chronic lymphocytic leukemia. Respir Med Case Rep 2015;16:131-3.

14. Teng CL, Li KW, Yu JT, et al. Malignancy-associated chylothorax: a 20-year study of 18 patients from a single institution. Eur J Cancer Care (Engl) 2012;21:599-605.

15. Eichhorst BF, Busch R, Stilgenbauer S, et al. First-line therapy with fludarabine compared with chlorambucil does not result in a major benefit for elderly patients with advanced chronic lymphocytic leukemia. Blood 2009;114:3382-91.

16. Brown JR. How I treat CLL patients with ibrutinib. Blood 2018;131:379-86.

17. Molica S, Matutes E, Tam C, et al. Ibrutinib in the treatment of chronic lymphocytic leukemia: 5 years on. Hematol Oncol 2020;38:129-36.

18. Gribben JG, Bosch F, Cymbalista F, et al. Optimising outcomes for patients with chronic lymphocytic leukaemia on ibrutinib therapy: European recommendations for clinical practice. Br J Haematol 2018;180:666-79.

Cite this article as: Cheng L, Huang M, Wei J, Xu H, Zhou M, Jiang L. Complete resolution of chylothorax with ibrutinib in chronic lymphocytic leukemia: a case report. Ann Palliat Med 2022;11(5):1833-1837. doi: 10.21037/apm-21-989 